\section{ANALYSIS OF THE ACCURACY OF VIRTUAL CLAMPING IN THE FIELD OF 3D SCANNING}

RADOMIR MENDRICKY, VLADIMIR KAFKA

Department of Manufacturing Systems and Automation, Technical University of Liberec, Liberec, Czech Republic

DOI: 10.17973/MMSJ.2021_03_2020068

e-mail: radomir.mendricky@tul.cz

When measuring large and flexible parts, specialised measuring and clamping jigs are used as the standard, which fix the part to the required position. However, because the production of such jigs is time consuming and very expensive, software tools have recently started to appear on the market, which allow even large flexible parts to be measured in a free state and to use so-called virtual clamping for alignment the part in the mounting position. This technique uses the finite element method to simulate deformations during clamping. In this way, the inspection time can be significantly reduced and costs can be saved. However, the question remains how reliable and accurate this method is compared to the measurements in a physical jig.

The article introduces the results of the analysis of the accuracy and repeatability of virtual clamping on the part "front fender of the Octavia car". The research showed that the conformity has not yet reached the values declared by the manufacturer, on the other hand, the measurement uncertainty during the virtual clamping was lower than during the measurement in a real jig. This, together with other indicators, indicates the great potential of the virtual clamping method in the future.

KEYWORDS

Virtual clamping, 3D digitisation, optical 3D scanner, 3D measurement, ATOS TripleScan, measuring jig, accuracy.

\section{INTRODUCTION}

Optical 3D digitisation is increasingly replacing coordinate measuring machine (CMM) measurements. Its popularity, in practice, is given not only by the speed of the measurement and the possibility of evaluating any demanding shape, but also due to the increasing accuracy of the measurement [Navrat 2019], [Mendricky 2015, 2016]. During 3D scanning, the part is first digitised and the inspection only takes place on the virtual model thus obtained. This allows, in addition to the classic dimensional measurement and Geometric Dimensioning and Tolerancing (GD\&T), a direct comparison of the part with the nominal CAD model. However, even with non-contact scanning, the part must be clamped to the desired position before the measurement. This is especially important for large and flexible parts (e.g., sheet metal stampings). Such a clamping most often simulates the positioning of the part in the assembly, i.e., it defines the position of the assembly points. The Reference Point System (RPS) is often used to align the part to the desired coordinate system. According to the defined reference points, all degrees of freedom are gradually removed from the part, in some cases, the secondary RPS points are determined, which also partially deform the part and give it the desired shape [Kafka 2020].
Therefore, it is necessary to provide a separate measuring jig for each type of inspected part. This is very costly, as in addition to the production, adjustment, debugging and testing of the jig, the price must include, for example, the costs of its development, operation, maintenance and storage [Johansson et al. 2017]. Some manufacturers of inspection software are, therefore, trying to come up with a new solution. One of them is a new functionality in the SW GOM Inspect Professional from the company GOM called Virtual Clamping (VC). It is a special module that solves the whole clamping process virtually in the software environment in order to completely replace mechanical clamping jigs. At first glance, this is an interesting solution that can significantly reduce the time and cost of inspection, on the other hand, the question is what accuracy, respective to the compliance with the classical measurement method, can be expected. Although the manufacturer declares certain reliability values, these are conditioned by many requirements (homogeneity of the material, size of the part, accurate knowledge of many physical constants of the material, etc.). Therefore, our own research was carried out, the intention of which was to assess this method on a real job and to show the possible pitfalls of virtual clamping. The main goal was to compare the degree of agreement of the virtual clamping in comparison with the classical method of measurement in a physical jig.

\section{CLAMPING WHEN MEASURING}

The clamping of the components in the measuring jig is most often undertaken with washers, clamps, mandrels, etc. There can be several reasons for clamping: fixation to a predetermined position due to the automation of the measuring cycle, the adjustment to the exact position within the assembly, due to flexibility of the part, residual stress or only to reduce the effect of gravity on the part. Because the task is to position the part as accurately as possible to the required points and, if necessary, to ensure its elastic deformation, it must be significantly stiffer than the measured part [Tuominen 2011].

\subsection{Positioning of the measured part}

In order to be able to compare the measured data with the nominal CAD model and evaluate the tolerances, it is necessary to align (register) the measured part (model) in a common coordinate system. The alignment of the measured and nominal part is realised by a 3D transformation including three translations and three rotations. The process can be divided into the alignment of rigid and flexible parts. A rigid part is a part that does not deform beyond its prescribed tolerances due to gravity or residual stress [Li 2004].

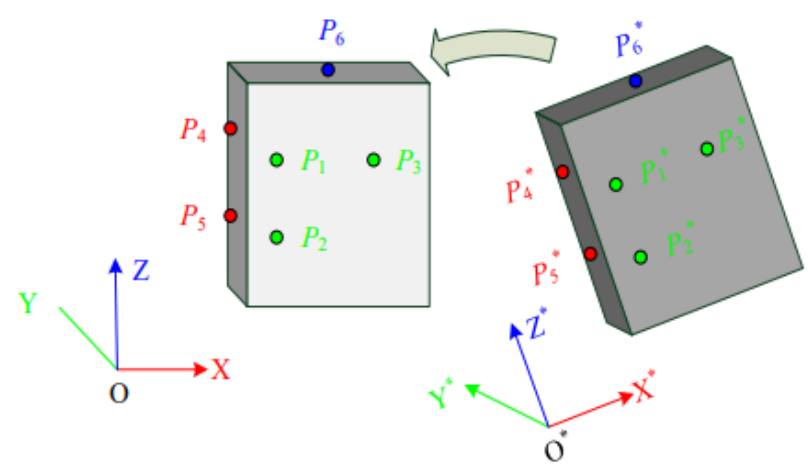

Figure 1. Alignment of the current part (right) to the nominal model (left) using RPS points [Rai 2016] 
In the automotive industry, so-called RPS points are often used for alignment. In this case, 6 basic RPS points are defined, thanks to which the registration to the nominal model is performed using the rule 3-2-1 (plane, line, point) and the transformation matrix is calculated - see Figure 1 [Rai 2016].

\section{Alignment of a rigid part}

In the process of setting up a rigid part, this is a disproportionately easier task compared to a flexible part, because the effects of gravity causing deformation on the measured part are so small that they can be neglected. Extensive research of the alignment has been described, for example, in the literature [Li 2004], which deals, among other things, with methods of describing the surfaces for the subsequent alignment of parts. The most common method of 3D alignment is ICP (Iterative Closest Point), first designed by Paul J. BesI [Besl 1992], from which many other improved variants of ICP are based. In this method, the Euclidean distance between two point clouds is calculated by the iterative processes of rotation and translation. This process does not require a shape representation of the surfaces, but requires a rough assignment of the surfaces to each other in order to define pairs of points at which the distances are minimised. The ICP method is often referred to as the Best-fit function [Li 2004]. Another method introduced by Jingfan Fan [Fan 2016] is 3PCHM (3-Points Convex Hull Matching). Briefly, this method first involves creating a convex envelope from a cloud of points, from which a random triangular mesh is generated. The lengths of the individual triangles are compared to create pairs between the measured and nominal triangles. All this is done iteratively, in order to find the maximum number of pairs. By minimising the distances between the vertices of an associated triangle, an ideal transformation is then created.

\section{Alignment of a flexible part}

When aligning a flexible part, it is not just a matter of finding the transformation matrix by minimising the distances between two point clouds. In order to be able to compare the measured part with nominal data, it is necessary to compensate for the elastic deformation or the inaccuracies caused by the technology of the production of the measured part. This can be achieved either by deforming the nominal CAD model or, conversely, the actual model itself.

In the literature [Abenhaim 2011], a single IDI (Iterative Displacement Inspection) method is proposed that does not use FEM analysis. IDI combines registration methods for rigid and flexible parts. The complete setup is undertaken in five main steps. First, the scanned part is roughly aligned with the CAD data, which then allows the use of the ICP method. When aligning with ICP, only those points that are not in the area of large deformations are aligned. The next step is to create an offset field to estimate the required deformation of the nominal CAD model for comparison of the scanned part (Figure 2). The purpose of this field is to represent the deformations caused by the clamping force, gravity and the manufacturing process only. By adding such a displacement field, the CAD model is deformed using affinity matrices and creates a new model that has the same deformation as the scanned part. Thanks to this, it is then possible to compare both parts.
CAD model

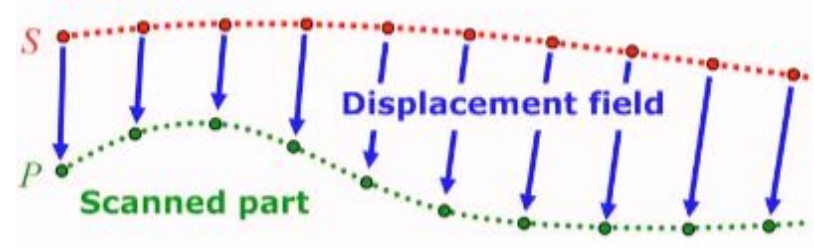

Figure 2. Deformation required by the CAD model to reflect a scanned part without a profile deviation [Abenhaim 2011]

In most cases, however, FEM simulations are used to calculate the required deformations of flexible parts, whose methods are called virtual clamping (VC).

\subsection{Virtual clamping (VC)}

The method using the deformation created on the measured part is dealt with, for example, by [Weckenmann 2006], where the deformation of the sheet metal part is simulated using the FEM method. In this work, the scanned cloud of the sheet metal points is converted to an STL format. The triangular mesh is then assigned a constant sheet thickness. Then a virtual clamping is performed. However, for the deformation analysis, the input information is not loading forces, but positions of the clamping points taking all degrees of freedom. Practically the same task is dealt with in [Gentilini 2011] with the difference that the plastic part is measured.

The method where the scanned part is not deformed when the measured part is set with the nominal CAD data, but with the CAD model, is dealt with the work of [Jaramillo 2009]. Again, the FEM method is used here. The clamping points are marked on both parts, but in the case of a scanned part, these points are located at different coordinates due to their deformation. The nominal CAD model is, therefore, deformed according to these points and the resulting FEM model is compared with the scanned data. However, the main aspect of this work is the use of the RBF (Radial Basis Function) method in order to reduce the time required for the FEM simulation due to the online inspection of the manufactured parts. Another work where nominal CAD data is used for the FEM simulation can be found in [Jaramillo 2013]. Unlike the previous work, the proposed method does not require a complete scan of the part, but only a part of its area to be inspected, in order to speed up the inspection. Here, too, the RBF method is used, albeit for a different purpose, when estimating the missing clamping points.

Another method introduced for the registration of flexible parts is the GNIF (Generalised Numerical Inspection Fixture) method. The main goal of this method is to replace the need for clamping jigs. In this method, the geodetic distance between two arbitrary points on the surface of the part is used, which does not change with the deformation. First, the measured part is placed on predefined supports and is scanned. Subsequently, an FEM simulation of the action of gravity is created on a nominal CAD model. It is then set in the same position with the support points and with the same orientation of the gravitational acceleration as the measured part. Subsequently, the set-up of the deformed CAD model and the scanned model using ICP is performed. The similarity is found for both of these models using a tool for measuring the similarity of the geodetic distances. Then one FEM model of the clamped CAD model is created and thanks to the geodetic similarity, a model of the clamped scanned part is also created. Finally, it is possible to compare both parts [RadvarEsfahlan 2012], (see Figure 3). 


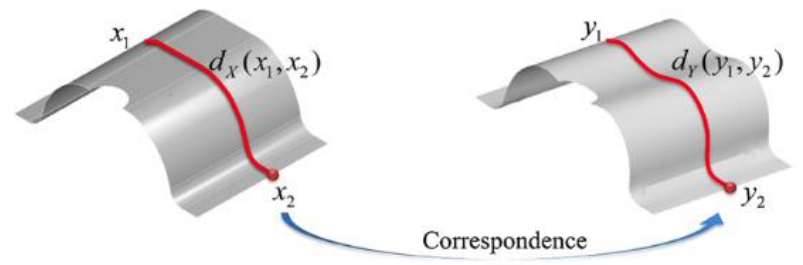

Figure 3. Simplified representation of the similarity measurement [Radvar-Esfahlan 2012]

The above research is mainly at the level of theoretical studies. Recently, however, GOM introduced a new functionality in the GOM Inspect Professional inspection software, called Virtual Clamping (available from version 2019). It is probably the first to fully integrate virtual clamping methods into a user software. However, the ability to virtually clamp a part is conditioned by the creation of a deformation model, which is a paid service of the GOM company.

However, as soon as the deformation model is available, it is possible to arbitrarily clamp the part virtually, to change the clamping parameters (number and positions of clamping points). The VC can be applied to rigid and flexible parts, whose deformation must not exceed the limit of elasticity. Furthermore, assemblies, parts made of composite materials and parts whose dimensions exceed $2 \mathrm{~m}$ cannot be simulated for the time being.

The inputs for the simulation are:

- Young's modulus

- Poisson's number

- Material density

- Measured model and position of the support points

- Nominal CAD data

- Position of the virtual clamping points

The detailed description of the VC method is the guarded knowhow of the company; however, it is clear from the available materials that this method uses the finite element method (FEM) to simulate the deformations caused by the physical clamping of the part. The inspected product is, therefore, scanned in a free state, where it is supported at any three points. The SW can then use FEM to read the deformations caused by gravity and then deform the part on the basis of virtually defined clamping points (see Figure 4).

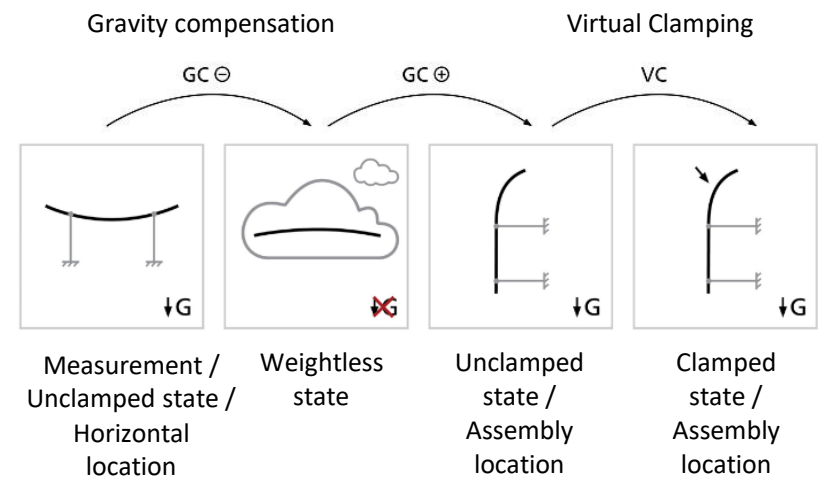

Figure 4. Principle of the gravity compensation and virtual clamping in SW GOM Inspect [GOM 2019]
Within this procedure, we can define 4 states - phases (hereinafter referred to as "STATE A - D"), with respect to three transition steps between these states (hereinafter referred to as "STEP 1 - 3") - see Figure 5.

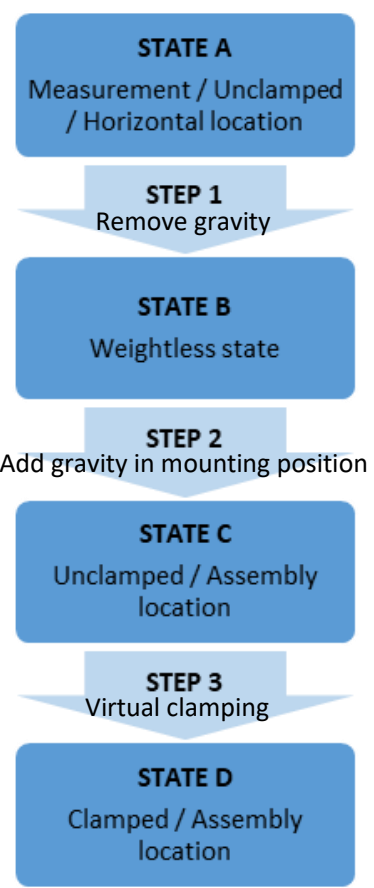

Figure 5. Individual phases and steps in the virtual clamping

\section{METHODS AND MATERIALS}

\subsection{Measured part}

For the purposes of the analysis, the left front fender from the Škoda Octavia A7 facelift (hereinafter referred to as the "fender") was selected, which meets all the criteria for creating a VC from GOM. It is a representative object, which, by default, must be clamped in the measuring jig during the measurement, as it is very flexible and, during the inspection, it is necessary to correct the production deformations and set the part in the position in which it will be mounted in the car assembly.

\subsection{Measuring system}

The ATOS III TripleScan measuring system was used to scan the part (Figure 6). It is an optical 3D scanner from the German manufacturer GOM, which works on the principle of triangulation and structured light. The whole system consists of a scanning head (scanner), which contains three lenses - a projection unit with a blue light and two cameras, as well as a control unit, a tripod and a powerful computer. By selecting the suitable camera and projector lenses, we define the size of the $3 \mathrm{D}$ area in the scanning direction in which the component can be digitised - the so-called measuring volume. This is crucial not only for the size of the scanned part, but it also significantly affects the density of scanned data and the accuracy of scanning itself. In our case, the MV $700(700 \times 530 \times 530 \mathrm{~mm})$ was used. The scanner was placed on the robot within the so-called Scan Box, so the whole scanning process was automated. The transformation of the individual images into a common coordinate system (into one unit) takes place on the basis of common reference points, which must be placed on the measured part or on the measuring jig before the measurement. 


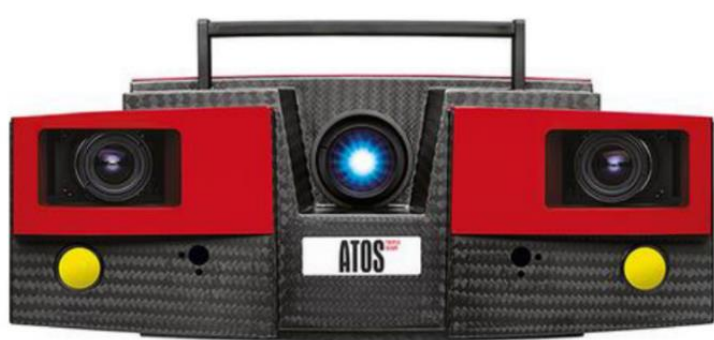

Figure 6. ATOS TripleScan optical 3D scanner [MCAE Systems 2020]

\subsection{Evaluation software}

The scanned data was processed in the SW GOM Inspect Professional $\vee 2019$. It is a program developed by GOM and is used to control the scanner, for the subsequent work and evaluation of the scanned data. When evaluating, the scanned part must first be set up with a nominal model. This can be performed in many ways, a Best-fit function or a alignment using the RPS points is most often used. Colour maps or sections can be used for the evaluation, various geometric elements can be measured, geometric tolerances can be evaluated, etc.

\subsection{Measurement procedure}

\section{Measurement in the jig}

The first step was performed in the standard way. The part was clamped according to the recommended procedure (instructions) in a special measuring jig produced for this part (Figure 7).

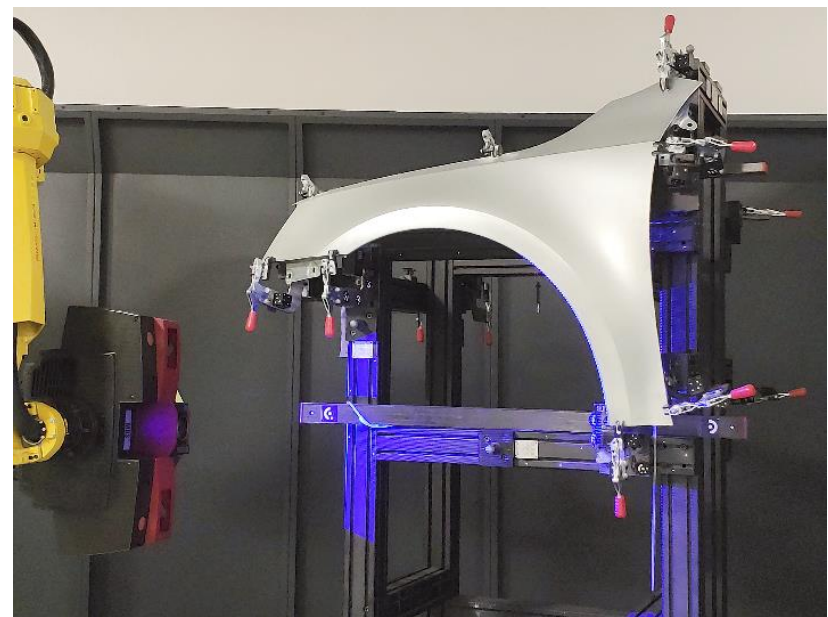

Figure 7. Scanning of the fender in a physical device

The jig contained 6 main RPS points (for positioning within the coordinate system) and 5 secondary RPS points (for the deformations simulating the clamping in the assembly). The jig also had three reference spheres, which represented the jig's own RPS system for determining its coordinate system within the car (see Figure 8).

The part was completely digitised, a polygonal mesh was calculated from the point cloud, which was exported to the STL model (hereinafter referred to as Model_R). One scan cycle took about 20 minutes. The whole process, including the new clamping, was repeated a total of 10 times.

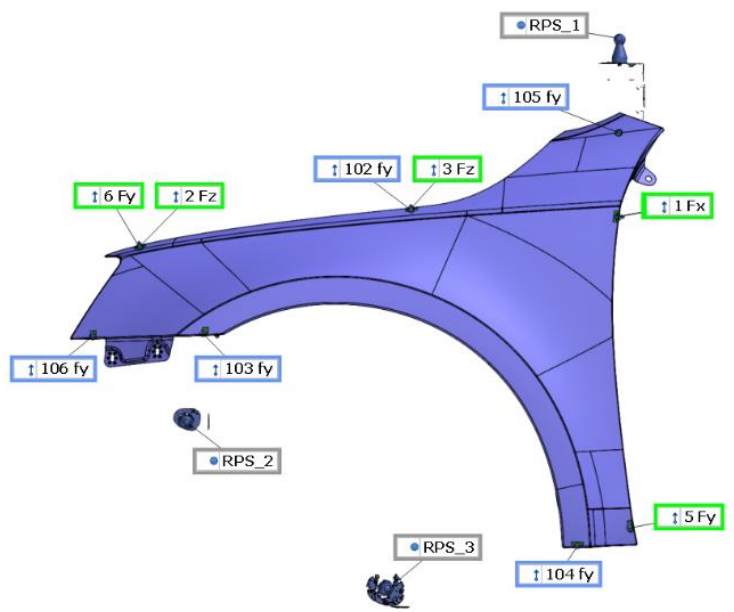

Figure 8. RPS points of the jig and fender

\section{Free-standing measurement and virtual clamping}

In the second step, the same part was measured by the virtual clamping method. The fender was scanned in a loose state. For this purpose, a simple jig with three support points was made, on which the fender was loosely placed (see Figure 9). The polygonal mesh in the STL format was again calculated from the individual scans. After scanning the fender, it was removed and the positions of the support points were measured (scanned). During the processing in the inspection SW, the procedure was carried out (with the help of a deformation model) in the individual steps (as shown in Figures 4 and 5).

STEP 1) Gravity subtraction acting on the part.

STEP 2) The model was virtually clamped - positioned in the functional position according to the main RPS points and the effects of gravity in this position were added.

Step 3) The part was virtually clamped to the remaining points, i.e., the secondary RPS points, and the deformation after this virtual clamping was calculated.

The resulting model, thus obtained, represents a virtually clamped part and will hereinafter be referred to as Model_VC. The whole process was repeated 10 times.

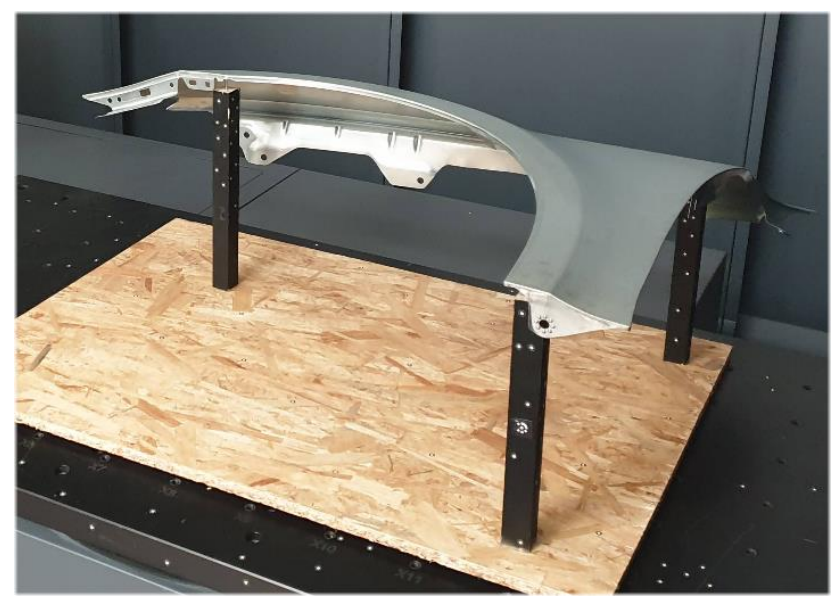

Figure 9. Scanning of the fender in the free state in a horizontal position (supported at three points) 
To measure a realistically and virtually clamped part, it is therefore necessary to:

- Scanning system (in our case ATOS III TripleScan)

- SW for scanning the control and subsequent inspection (in our case, GOM Inspect Professional in 2019)

- Inspected part (in our case, the fender)

- Nominal CAD model of the inspected part

In addition, for measurements in a real jig, we need:

- Measuring jig for the given part type (all clamping RPS points correctly adjusted)

In addition, to measure a virtually clamped part, we need:

- Device for measuring in the free state

- Coordinates of clamping RPS points

- Deformation CAD model

- Material properties of the part (density $(7800 \mathrm{~kg} / \mathrm{m})$, Poisson's ratio (0.3), Young's modulus (207 $000 \mathrm{MPa}$ )
After obtaining the current models in both ways, the analysis of the data was performed. This consisted in the comparison of the models obtained by the measurements in the real jig (Model_R) and the models obtained by the measurements in the free state after their virtual clamping (Model_VC). For a better validation of the results, the repeatability of the scan itself was verified before this analysis. This consisted in aligning the scans from the real fixture to the nominal data according to the RPS of the fixture spheres (their position should be stable and unchanged) and calculating the trend of the deviations of these RPS points. It turned out that, for all ten measurements, the maximum deviation between the individual measurements at the RPS points of the jig (ball centres) is up to $0.02 \mathrm{~mm}$ (see Figure 10), which is considered satisfactory and corresponds to the stated accuracy of the scanning system.

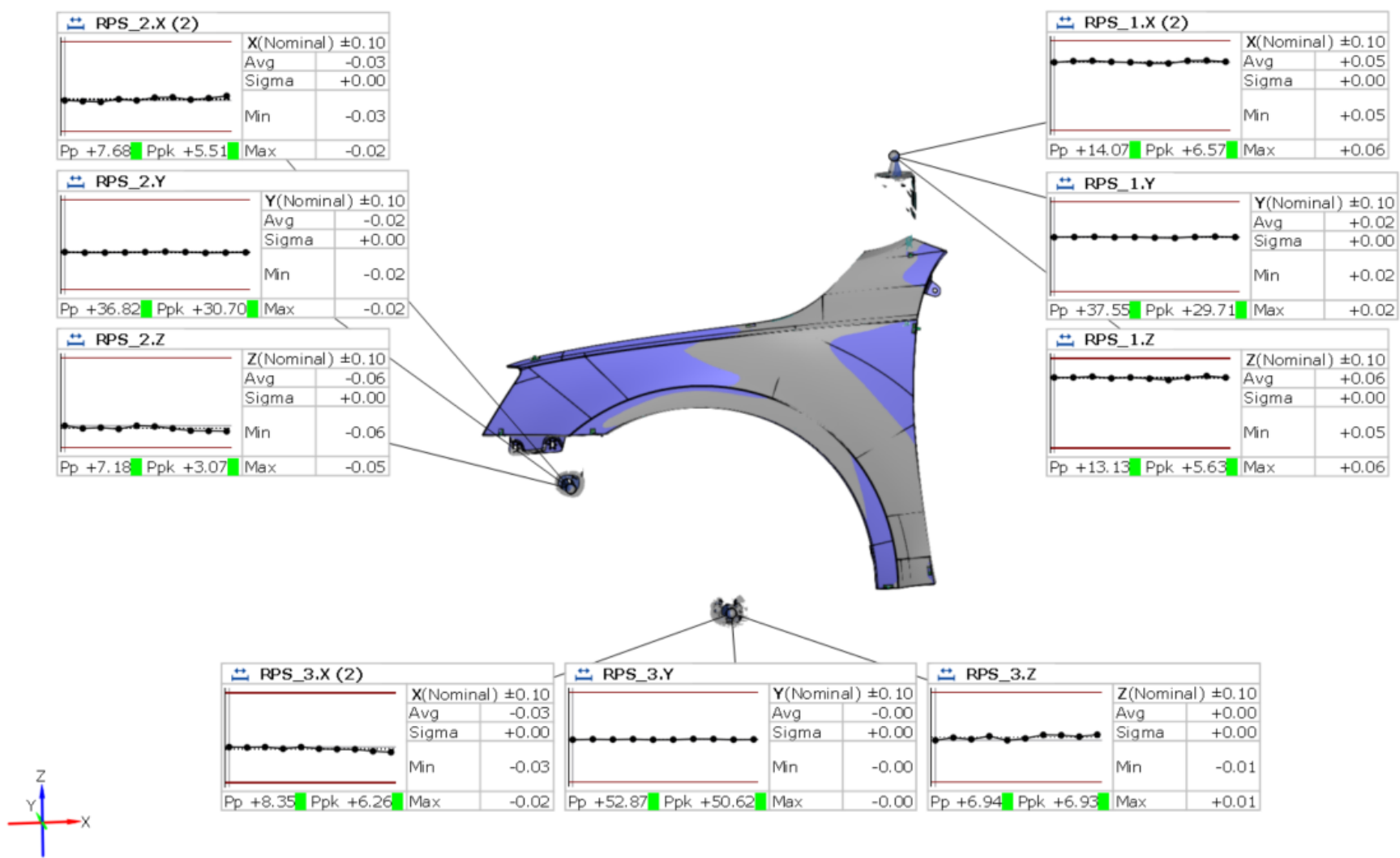

Figure 10. The set up on the RPS jig, the trend of the repeatability of measurements

The above trend analysis is used to monitor and evaluate the measured data for similar or the same objects. Each trend displays the following information.

- Name of the measured element (e.g., RPS_2.X)

- Type of the measured element and tolerance (e.g., $X$ (Nominal) \pm 0.10 )

- Average deviation from the nominal value (e.g., AVG 0.03)

- Standard deviation (e.g., Sigma +0.003)

- Minimum deviation (e.g., Min -0.03)

- Maximum deviation (e.g., Max-0.02)

- Pp value - expresses the performance of the process and how the measured element meets the requirement for fluctuations within the tolerance, but does not take its position into account (e.g., $P p+7.68)$

- Ppk value - expresses the performance of the process and how close it is to the relevant specification limits (e.g., Ppk+5.51)

\section{RESULTS AND DISCUSSION}

For better illustration and visualisation of what is happening with the model and what change of shape, with respect to the deformation, occurs after the application of the partial steps of virtual clamping, a colour map of deviations is always calculated between the individual states (STATE A-D). This is shown in the following figures for all three steps (STEP 1-3). Each colour map, therefore, expresses the differences in the shape of the part (normal deviations) between the successive states, i.e., the effects of the individual steps. 


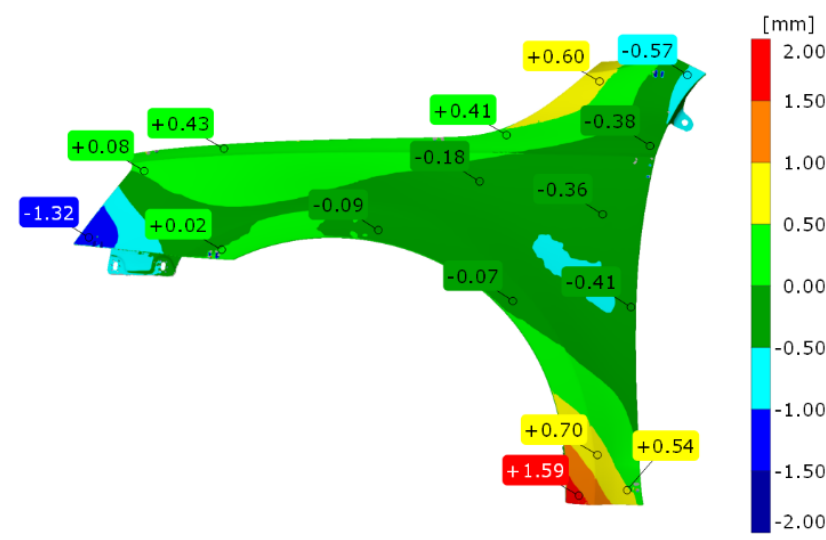

Figure 11. Colour map of the deviations / STEP 1 (alignment by Best-fit)

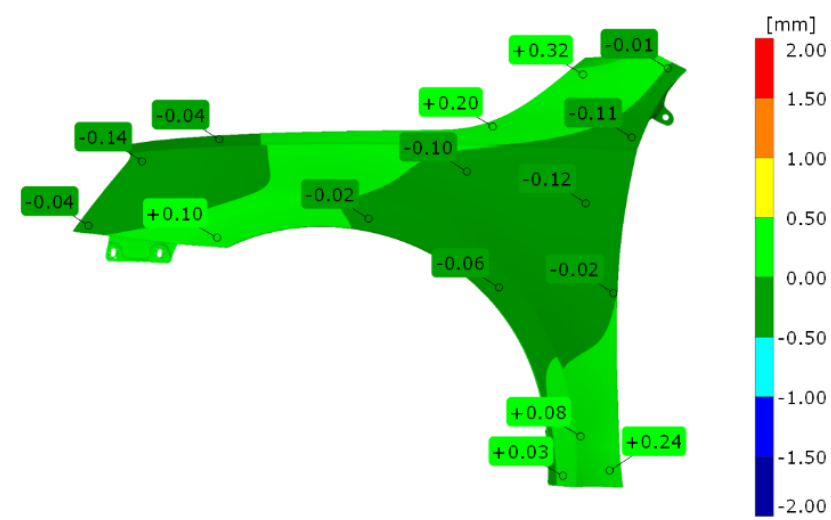

Figure 12. Colour map of the deviations / STEP 2 (alignment by Best-fit)

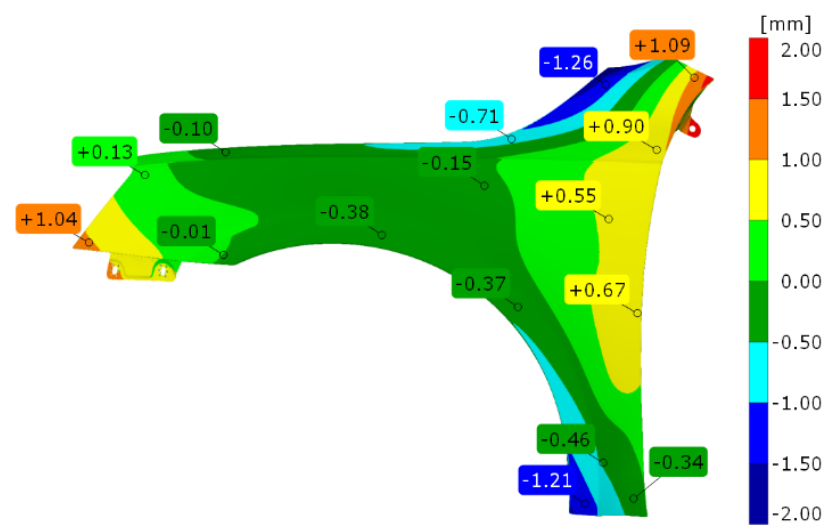

Figure 13. Colour map of the deviations / STEP 3 (alignment by Best-fit)

From these deviation maps, it is clear, at first glance, that the most significant deformation of the part is by its own gravity (subtraction of the gravity changed shape with the maximum absolute values around $1.5 \mathrm{~mm}$ - see Figure 11 ) and clamping the part to the secondary RPS points (where the deformations take on similar values (see Figure 13)). On the contrary, the effects of gravity on the part in the vertical position and its positioning in the main RPS points have a minimal effect on the shape of the part. The deviations caused by this step (see Figure 12) are a absolute maximum of $0.3 \mathrm{~mm}$.

By default, the colour maps are used primarily to detect manufacturing errors, i.e., to compare the scan of the real part against the CAD model. In our case, this information is not authoritative for us, because we do not evaluate the quality of the production, but we want to compare what will the deviations between the physically and virtually clamped part be - that is, the deviations of Modelu_VC_no. from Modelu_R_no. According to the GOM documents, this compliance should ideally reach a maximum deviation of $\pm 0.05 \mathrm{~mm}$ [GOM 2019]. The result of our measurements for the first checked pair is shown in Figure 14.
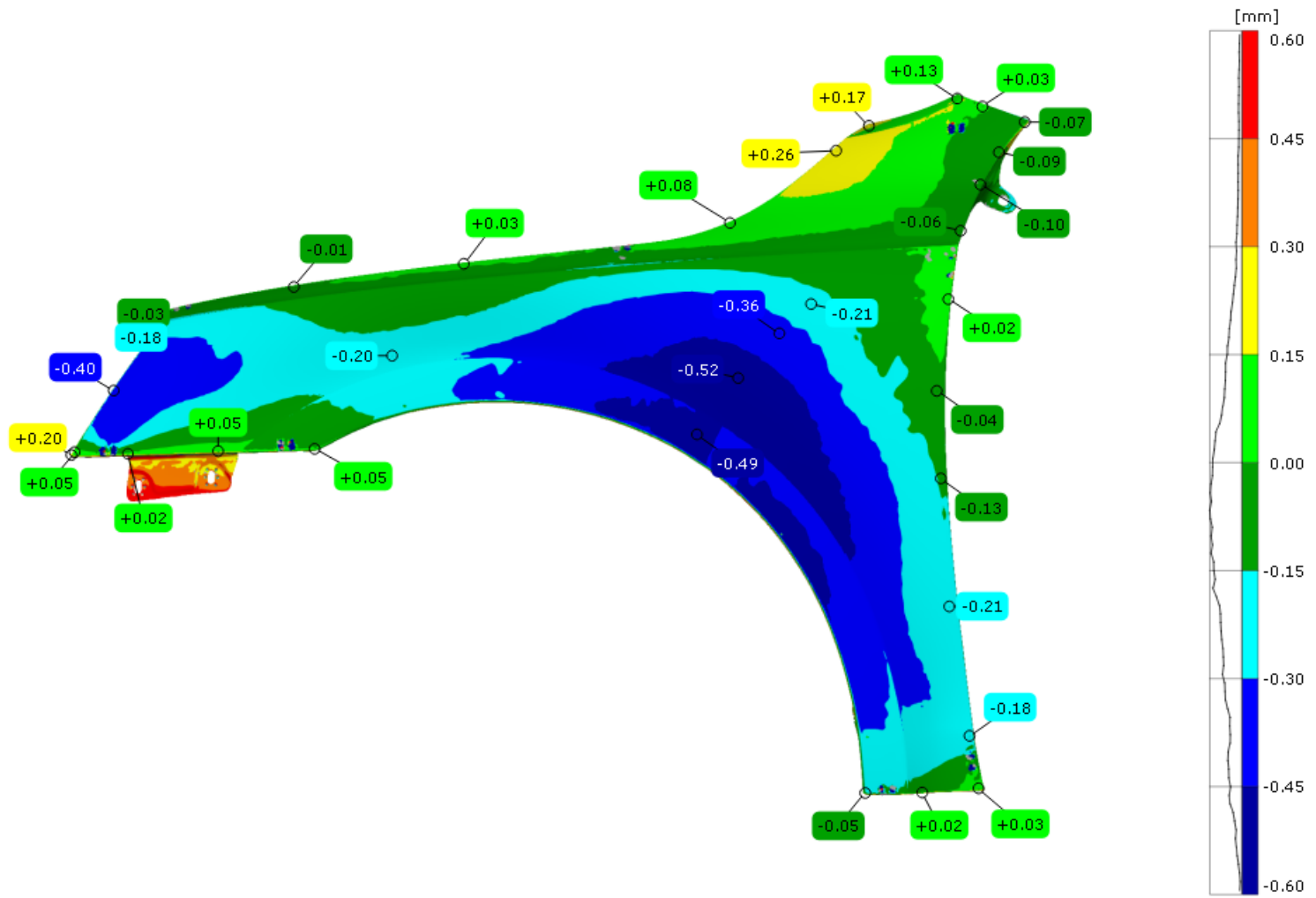

Figure 14. Deviation colour map - virtual clamping (Model_VC_1) vs. physical clamping (Model_R_1) (alignment by RPS) 
Similar colour maps were obtained from another nine comparisons. It is clear from the results that the agreement is not what would be expected and the deviations between the virtually and physically clamped part are, in extreme cases, up to about $\pm 0.5 \mathrm{~mm}$. Therefore, the ideal values stated by GOM were not reached. However, the actual state can be affected by many variables, such as the possible inaccuracies in the physical properties of the material, the correctness of the adjustment of the mechanical device, its insufficient rigidity, improperly set parameters during simulations, etc.

The most controlled dimensions of the visible sheets metal are the positions of the edges, as their accuracy affects the dimensions of the joints and the fit between the individual car body parts. To compare the accuracy of the mechanical and virtual clamping, deviation trends from all 10 measurements were created on a colour map where the fender aligns with the bonnet, light, door, front bumper, thresholds and A-pillar - see the example in Figure 15.

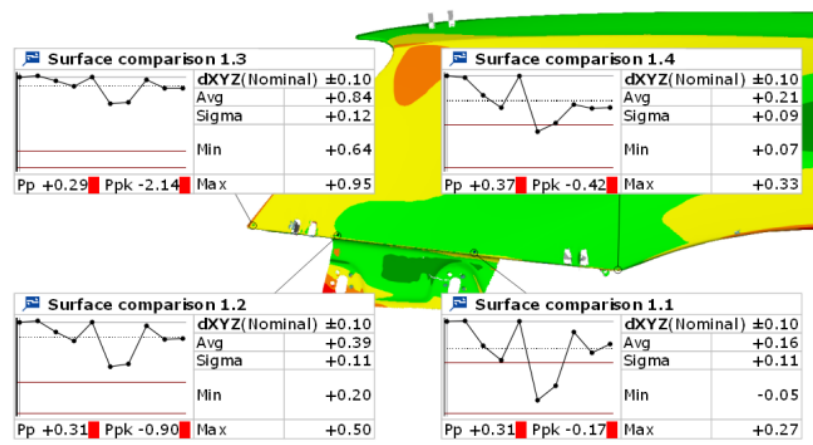

Figure 15. Example of the trend analysis in the area of the front bumper - physical device vs. CAD (alignment by RPS)

These trends, namely the Standard Deviation and the Range (which indicate the difference between the maximum and minimum deviation), were evaluated for all the monitored sites, both in terms of the analysis of the part in the physical jig vs. the $C A D$ one, both from the analysis of the virtual clamping vs. the real product (see Table 1 ).

From this analysis, it is clear that the repeatability of the measurement is better (a smaller standard deviation) in the case of the virtual clamping. Conversely, when clamped in a physical $\mathrm{jig}$, the results of the individual measurements often differed significantly. The detail of the colour map is illustrated at the top of the fender at the A-pillar in Fig. 16 and Fig. 17, respectively. Here, we can assess the differences in the measurement results of the part in the physical jig for the 5th, and 7th measurement, respectively. It can be seen from the figures that although this is the output of measuring the same part in the same fixture, the results differ. The total average of all the monitored values (last row of the table) is about half for both the range and the standard deviation in the case of the VC.

\begin{tabular}{|c|c|c|c|c|}
\hline & \multicolumn{2}{|c|}{ Real jig vs. CAD } & \multicolumn{2}{|c|}{$\begin{array}{l}\text { Virtual clamping vs. } \\
\text { real jig }\end{array}$} \\
\hline 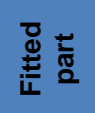 & $\begin{array}{l}\text { Range } \\
{[\mathrm{mm}]}\end{array}$ & $\begin{array}{c}\text { Standard } \\
\text { deviation } \\
{[\mathrm{mm}]}\end{array}$ & $\begin{array}{l}\text { Range } \\
{[\mathrm{mm}]}\end{array}$ & $\begin{array}{c}\text { Standard } \\
\text { deviation } \\
{[\mathrm{mm}]}\end{array}$ \\
\hline \multirow{4}{*}{ 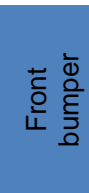 } & 0.31 & 0.12 & 0.07 & 0.02 \\
\hline & 0.30 & 0.11 & 0.11 & 0.04 \\
\hline & 0.22 & 0.09 & 0.2 & 0.07 \\
\hline & 0.26 & 0.11 & 0.07 & 0.03 \\
\hline Avg. & 0.27 & 0.11 & 0.11 & 0.04 \\
\hline \multirow{4}{*}{ 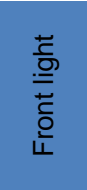 } & 0.03 & 0.01 & 0.18 & 0.06 \\
\hline & 0.09 & 0.04 & 0.16 & 0.06 \\
\hline & 0.21 & 0.07 & 0.15 & 0.05 \\
\hline & 0.31 & 0.11 & 0.03 & 0.01 \\
\hline Avg. & 0.16 & 0.06 & 0.13 & 0.05 \\
\hline \multirow{6}{*}{ 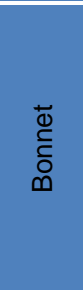 } & 0.03 & 0.01 & 0.03 & 0.01 \\
\hline & 0.03 & 0.01 & 0.05 & 0.02 \\
\hline & 0.02 & 0 & 0.09 & 0.03 \\
\hline & 0.14 & 0.06 & 0.08 & 0.03 \\
\hline & 0.40 & 0.16 & 0.07 & 0.02 \\
\hline & 0.34 & 0.14 & 0.22 & 0.07 \\
\hline Avg. & 0.16 & 0.06 & 0.09 & 0.03 \\
\hline \multirow{3}{*}{$\frac{1}{<\frac{\bar{\sigma}}{\bar{a}}}$} & 0.55 & 0.23 & 0.3 & 0.11 \\
\hline & 0.12 & 0.04 & 0.08 & 0.04 \\
\hline & 0.17 & 0.05 & 0.16 & 0.06 \\
\hline Avg. & 0.28 & 0.11 & 0.18 & 0.07 \\
\hline \multirow{3}{*}{ 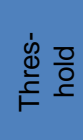 } & 0.09 & 0.03 & 0.07 & 0.03 \\
\hline & 0.38 & 0.14 & 0.2 & 0.06 \\
\hline & 0.21 & 0.06 & 0.15 & 0.05 \\
\hline Avg. & 0.23 & 0.08 & 0.14 & 0.05 \\
\hline \multirow{10}{*}{$\begin{array}{l}\overline{0} \\
\text { 음 } \\
\text { 든 } \\
\text { 눈 }\end{array}$} & 0.17 & 0.05 & 0.16 & 0.06 \\
\hline & 0.21 & 0.03 & 0.11 & 0.04 \\
\hline & 0.51 & 0.08 & 0.12 & 0.05 \\
\hline & 0.10 & 0.21 & 0.17 & 0.06 \\
\hline & 0.10 & 0.04 & 0.1 & 0.03 \\
\hline & 0.11 & 0.04 & 0.1 & 0.04 \\
\hline & 0.03 & 0.01 & 0.06 & 0.02 \\
\hline & 0.16 & 0.07 & 0.07 & 0.02 \\
\hline & 0.31 & 0.12 & 0.05 & 0.02 \\
\hline & 0.06 & 0.02 & 0.07 & 0.03 \\
\hline Avg. & 0.18 & 0.07 & 0.10 & 0.04 \\
\hline $\begin{array}{l}\text { Total } \\
\text { Avg. }\end{array}$ & 0.20 & 0.08 & 0.12 & 0.04 \\
\hline
\end{tabular}

Table 1. Ranges and standard deviations for the matched parts 


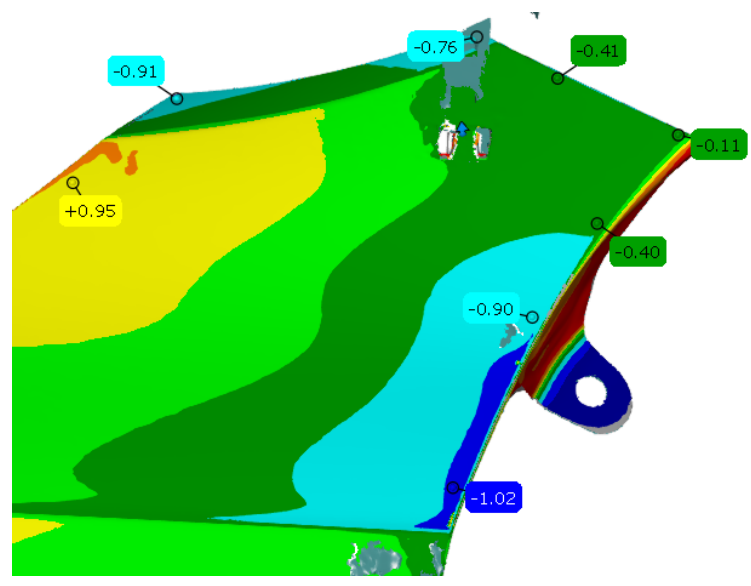

Figure 16. Detail of the colour map of the deviations - current model in the physical jig vs. CAD (Model_R_5 vs. CAD), (alignment by RPS points)

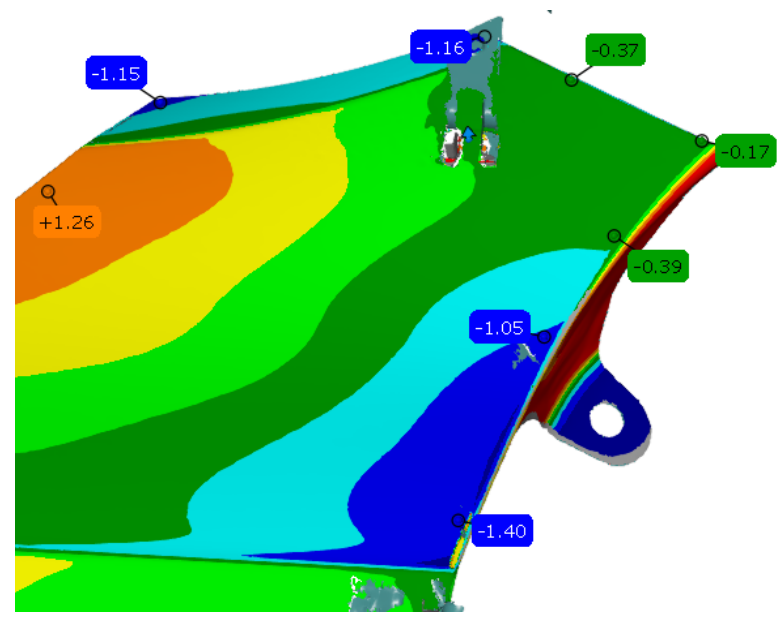

Figure 17. Detail of the colour map of the deviations - current model in the physical jig (Model_R_7 vs. CAD), (alignment by RPS points)

The poor repeatability when measured in the physical jig can be caused by many influences. The main thing will probably be the clamping itself, which is not $100 \%$ repeatable due to the insertion into the jig, the friction on the contact surfaces of RPS points, the tightening with clamps. Even small deviations in the foundation and alignment of the part will bring systematic errors to the results, which increases the standard deviation and measurement uncertainty.

All the colour maps that express the deviations between the virtual and real clamping, as well as the graphs expressing the frequency of the deviations within the whole part (see Figure 14 - right part of the picture, see Figure 18 for details on the first 5 measurements) show a very similar pattern. Most deviations are in the range from $+0.15 \mathrm{~mm}$ to $-0.3 \mathrm{~mm}$. The largest deviations are recorded in the central area of the mudguard at the wheel arches and extend from the lower edge of the door through the centre of the mudguard to the area of the lights. The maximums reach $0.6 \mathrm{~mm}$ in the area of the wheel arches. It is clear from this that the largest deviations are found in places with the absence of RPS points. The problems with the headlights are probably due to the fact that points $2 \mathrm{Fz}$ and $6 \mathrm{Fy}$ are located above the tornado line, through which they are not able to deform the area below it. Thus, RPS $106 \mathrm{fy}$ is the only point that helps maintain the headlight area, which may be the reason for such large deviations at this location.

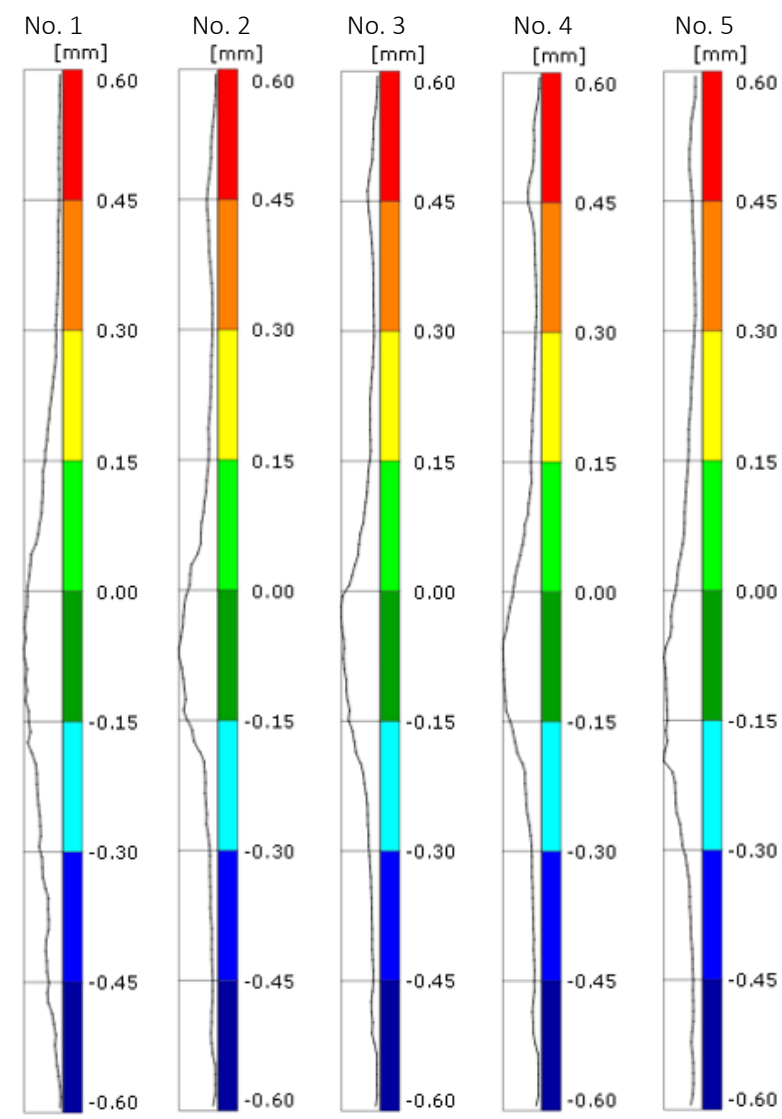

Figure 18. Histogram (frequency of representation) of deviations - virtual clamping (Model_VC) vs. physical clamping (Model_R), measurement No. 1 - 5 (alignment by RPS)

The situation is statistically better in the area of key mating parts, where the deviations are smaller due to the controlled deformation of the RPS points located here, than, for example, in the mentioned centre of the fender or at the headlights. The graph in Figure 19 expresses the quadratic mean of the deviations from all ten measurements. The individual labels are arranged sequentially in a clockwise direction, from the area of the front bumper to the area of the threshold under the front door.

The graph shows large deviations in the area of the front bumper, which are not clearly visible from the colour maps, because they are located close to the edge. Furthermore, the problem place for the headlights below the tornado line, where the quadratic mean of the deviations is $0.23 \mathrm{~mm}$, is confirmed. The bonnet achieves very small deviations in the range of 0.03 $\mathrm{mm}$ to $0.09 \mathrm{~mm}$, except in the hinge area, where the average deviation is $0.25 \mathrm{~mm}$. Even in this case, it is a place between two RPS points $3 \mathrm{Fz}$ and $105 \mathrm{Fy}$. In the door area, most deviations are around $0.15 \mathrm{~mm}$, except for labels no. 22 and no. 23. These are located below the tornado line near RPS $1 \mathrm{Fx}$ and $4 \mathrm{Fy}$ and label no. 27 at the thresholds for RPS $5 \mathrm{Fy}$, where the average deviations do not exceed $0.07 \mathrm{~mm}$. For the A-pillar, the largest average deviation is at the edges of the line, which is located at the greatest distance from the RPS $105 \mathrm{Fy}$. The best results are obtained in areas of the lower threshold, where no average quadratic deviation exceeds $0.05 \mathrm{~mm}$. 


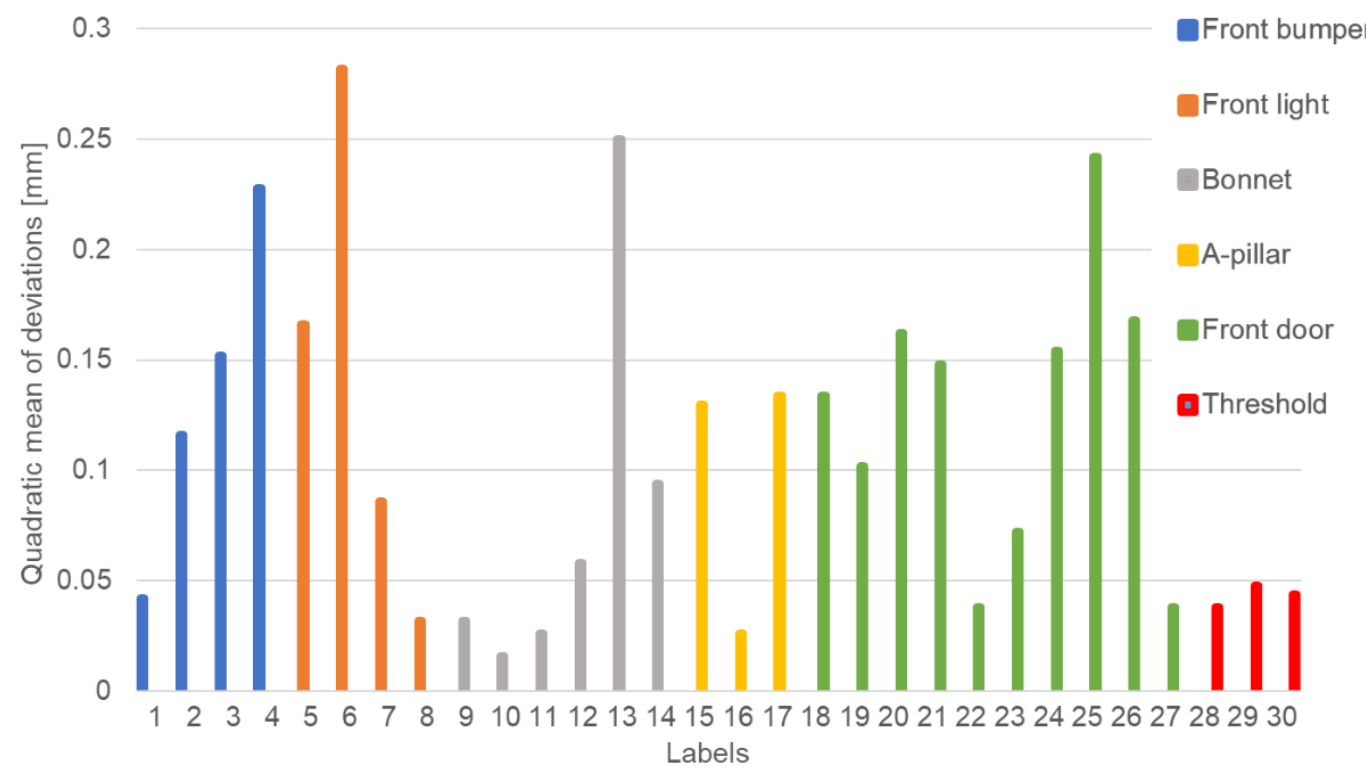

Figure 19. The quadratic mean of the deviations of the individual labels of the matched parts - virtual clamping (Model_VC) vs. the physical clamping (Model_R) (alignment by RPS)

After evaluating all the results, it is possible to say the following about the VC.

- In the case of our research, the VC did not reach an accuracy of $\pm 0.05 \mathrm{~mm}$, as stated by GOM.

- At the location of the mating parts, the deviations between the VC and the measurement in the physical jig were approx. $\pm 0.3 \mathrm{~mm}$, and within approx. $\pm 0.6 \mathrm{~mm}$ for the whole part.

- The VC achieved better repeatability of measurements, in the real jig, almost twice as good as the absolute deviations from the mean value were achieved.

- Overall, the VC can be considered the better results. For a real jig, it is necessary to digitise the fender several times to evaluate the measurement to eliminate the clamping error.

\section{CONCLUSIONS}

The aim of the research was to evaluate a new method of Virtual Clamping from the German company GOM. The purpose of this method is to replace the mechanical clamping of parts during measurement using a mathematical simulation. The verification was performed on the sheet metal moulding of the front door of a car. All measurements (in the physical jig, the virtual clamping) were repeated 10 times.

A comparative analysis of the parts scanned in the real jig and the virtually clamped models showed that the match did not reach the declared error of $\pm 0.05 \mathrm{~mm}$, but the deviations between the VC and the measurement in the physical jig were approx. $\pm 0.3 \mathrm{~mm}$ at the location of the mating parts, and within approx. $\pm 0.6 \mathrm{~mm}$ for the whole part. VC achieved the worst results in the areas without any significant indentations that would increase the rigidity, and in areas with the absence of clamping points. However, due to the high measurement uncertainty found in the real jig, these deviations can be partly attributed to the fact that the data from the measurements in the jig, which was to serve as a reference, did not show good stability. The repeatability of the virtual clamping was about twice as good as the repeatability of the measurements in the physical jig.
Despite the not entirely convincing results, it can be said that the method of virtual clamping has proven to be precise enough to replace jigs in certain cases. To increase the accuracy and unquestionability of the results, it is necessary to ensure the correct setting of the simulation and to know the exact material properties of the measured part. Undoubtedly, the economic aspect will also be a great motivation for companies, as the cost of a physical jig for such large parts can be in the range of 10,000 - 30,000 Euros, the costs of VC are only the fees for the deformation model, which are currently 1,500 Euro. With this model, it is then possible to clamp virtually, to change the positions and the number of clamping points in any way.

Among the other advantages of VC include [GOM 2019]:

\section{Cost reduction}

- No individual fixture needed

- Less multiple measurements (e.g. clamped/unclamped)

- Simple change management (e.g. without mechanical changes)

- Early integration into process chain

- Reduction of optimization loops

\section{High process capability}

- Perfect accessibility for optical measurements

- Mathematically perfect boundary conditions

- No undefined friction at the clamps

- Reduced user influence

\section{New possibilities}

- Determination of clamping forces at each clamping point

- Clamped measurements in CT

It is difficult to evaluate VC from measuring only one part. Although the results indicate the great potential of this method, in the next research phases, it will be necessary to measure a larger portfolio of parts both in terms of the size (small, large), stiffness (rigid, flexible) and material (metal, aluminium alloys, plastic). Furthermore, it will be appropriate to focus the research into the influence of the process parameters (physical constants of the material, simulation parameters) on the accuracy of the virtual clamping simulation. 


\section{ACKNOWLEDGMENTS}

This work was supported by the Student Grant Competition of the Technical University of Liberec under the project Optimization of manufacturing systems, 3D technologies and automation No. SGS-2019-5011.

\section{REFERENCES}

[Abenhaim 2011] Abenhaim, Gad N. et al. A Novel Approach for the Inspection of Flexible Parts Without the Use of Special Fixtures. Journal of Manufacturing Science and Engineering, February 2011, Vol.133, No.011009-1., pp 1-11. ISSN 1087-1357

[Besl 1992] Besl, P. J. and Neil D. Mckay. A method for registration of 3-D shapes. IEEE transactions on pattern analysis and machine intelligence, February 2011, Vol.14, No.2., pp 239256. ISSN 0162-8828

[Fan 2016] Fan, J. et al. 3-Points Convex Hull Matching (3PCHM) for fast and robust point set registration. Neurocomputing, June 2016, Vol.194, pp 227-240. ISSN 0925-2312

[Gentilini 2011] Gentilini, I. and K. Shimada. Predicting and evaluating the post-assembly shape of thin-walled components via 3D laser digitization and FEA simulation of the assembly process. Computer-aided design, March 2011, Vol.43, No.3., pp 316-328. ISSN 0010-4485

[GOM mbH 2019] GOM mbH. Virtual Clamping.

[Jaramillo 2009] Jaramillo, Andrés E. et al. On-line 3-D inspection of deformable parts using FEM trained radial basis functions. In: 2009 IEEE 12th International Conference on Computer Vision Workshops, ICCV Workshops, Kyoto, Japan, November 2009. pp 1733-1739. ISBN 978-1-4244-4441-0

[Jaramillo 2013] Jaramillo, A. et al. Fast dimensional inspection of deformable parts from partial views. Computers in Industry, December 2013, Vol.64, No.9., pp 1076-1081. ISSN 0166-3615

[Johansson 2017] Johansson, Ch. et al. Product-Service Systems for Functional Offering of Automotive Fixtures: Using Design Automation as Enabler. Procedia CIRP, 9th CIRP IPSS Conference: Circular Perspectives on PSS, January 2017, Vol.64, pp 411-416. ISSN 2212-8271

[Kafka 2020] Kafka, V. Verification of a new method of virtual clamping in the field of 3D measurement / thesis. Liberec, Technical University of Liberec, Department of Mechanical Engineering, 2020. (in Czech).

\section{CONTACTS:}

Ing. Radomir Mendricky, Ph.D.

Technical University of Liberec

Faculty of Mechanical Engineering

Department of Manufacturing Systems and Automatization

Studentska 2, 46117 Liberec 1, Czech Republic

+420485353356

radomir.mendricky@tul.cz

www.ksa.tul.cz
[Li 2004] Li, Y. and P. Gu. Free-form surface inspection techniques state of the art review. Computer-Aided Design, November 2004, Vol.36, No.13., pp 1395-1417. ISSN 0010-4485

[MCAE Systems 2020] MCAE Systems, Products / ATOS Triple Scan. [online]. [cit. 08-10-2020].]. Available from < https://www.mcae.cz/produkty/atos-triple-scan/ >.

[Mendricky 2015] Mendricky, R. Analysis of Measurement Accuracy of Contactless 3D Optical Scanners. MM Science Journal, October 2015, Vol. 2015, No. October., pp 711-716. ISSN 18031269, 18050476

[Mendricky 2016] Mendricky, R. Determination of Measurement Accuracy of Optical 3D Scanners. MM Science Journal, December 2016, Vol.2016, No.6., pp 1565-1572. ISSN 18031269, 18050476

[Navrat 2019] Návrat, J. Comparison of measuring methods for quality control of parts / thesis. Ostrava, Technical University of Ostrava, Department of Machining, Assembly and Engineering Metrology, 2019. (in Czech).

[Radvar-Esfahlan 2012] Radvar-Esfahlan, H. and S.-A. Tahan. Nonrigid geometric metrology using generalized numerical inspection fixtures. Precision Engineering, January 2012, Vol.36, No.1., pp 1-9. ISSN 0141-6359

[Rai 2016] Rai, B. and L. Shenglan. RPS Alignment of Automotive Body Parts in Virtual Assembly and Deviation Analyses. International Journal of Scientific \& Engineering Research, September 2016, Vol.7, No.9., pp 562-570. ISSN 2229-5518

[Tuominen 2011] Tuominen, V. Virtual clamping in automotive production line measurement. Expert Systems with Applications, November 2011, Vol.38, No.12., pp 15065-15074. ISSN 0957-4174

[Weckenmann 2006] Weckenmann, A. and J. Weickmann. Optical inspection of formed sheet metal parts applying fringe projection systems and virtual fixation. Metrology and Measurement Systems, January 2006, Vol.13, No.4., pp 221334. ISSN 0860-8229 